\title{
Technical note: An in vivo method to determine kinetics of unsaturated fatty acid biohydrogenation in the rumen
}

\author{
M. Baldin, ${ }^{\star 1}$ D. E. Rico,† M. H. Green, $\ddagger$ and K. J. Harvatine ${ }^{\star 2}$ \\ *Department of Animal Science, Penn State University, University Park 16802 \\ †Department of Animal Science, Université Laval, Québec, QC, Canada G1V 0A6 \\ ‡Department of Nutritional Sciences, Penn State University, University Park 16802
}

\section{ABSTRACT}

Rumen microbial biohydrogenation $(\mathrm{BH})$ of unsaturated fatty acids (UFA) has been extensively studied in vitro; however, in vitro $\mathrm{BH}$ pathways, rates, and extents may not parallel those in vivo. The objective was to develop an assay to assess in vivo rates, pathways, and extent of $\mathrm{BH}$ of oleic (OA), linoleic (LA), and $\alpha$-linolenic (ALA) acids. Each UFA was characterized in a separate experiment, each using 4 ruminally cannulated lactating Holstein cows. A single bolus consisting of $200 \mathrm{~g}$ of a UFA-oil [experiment 1 (EXP1): $87 \%$ OA sunflower, experiment 2 (EXP2): $70 \%$ LA safflower, and experiment 3 (EXP3): 54\% ALA flaxseed] and 12 $\mathrm{g}$ of heptadecanoic acid (C17:0) was mixed into the rumen through the fistula. Rumen digesta was collected at $-1,-0.25,0.1,0.25,0.5,0.75,1,1.5,2,3,4$, and 6 $\mathrm{h}$ relative to the bolus. Overall, the triglyceride boluses increased total fatty acids (FA) in the rumen from 3.9 (standard deviation $= \pm 1.4)$ to $7.3 \%( \pm 1.4)$ of rumen dry matter and enriched C17:0 from $0.4( \pm 0.1)$ to $2.5 \%$ $( \pm 0.5)$ of FA. The bolus enriched OA from $8.9( \pm 1.0)$ to $30.1 \%( \pm 4.6)$ of FA in EXP1, LA from $11.1( \pm 1.8)$ to $35.9 \%( \pm 5.0)$ of FA in EXP2, and ALA from $2.1( \pm 0.1)$ to $19.8 \%( \pm 4.3)$ of FA in EXP3. The disappearances of C17:0, OA, LA, and ALA were fit to a single exponential decay model. The first-order rate of C17:0 rumen disappearance (turnover) was $9.1,6.9$, and $5.2 \% / \mathrm{h}$ in EXP1, EXP2, and EXP3, respectively, and was used as a marker of FA passage. The rate of total rumen turnover of OA was $54.1 \% / \mathrm{h}$, LA was $60.5 \% / \mathrm{h}$, and ALA was $93.0 \% / \mathrm{h}$ in EXP1, EXP2, and EXP3, respectively. Rumen concentration of all 3 UFA reached prebolus concentrations within $4 \mathrm{~h}$. The calculated extent of lipolysis and initial isomerization was $85.6 \%$ for OA,

Received July 6, 2017.

Accepted December 14, 2017.

${ }^{1}$ Current address: Provimi North America, 10 Nutrition Way, Brookville, OH 45309.

${ }^{2}$ Corresponding author: kjh182@psu.edu
89.8\% for LA, and $94.7 \%$ for ALA in EXP1, EXP2, and EXP3, respectively. Assuming that BH equals total disappearance minus passage, the rates of lipolysis and initial isomerization were $45.0,53.6$, and $87.8 \% / \mathrm{h}$ for OA, LA, and ALA in EXP1, EXP2, and EXP3, respectively. Analysis of the data using compartmental modeling showed that the normal $\mathrm{BH}$ pathways proposed in the literature explained 46.0, 37.3, and $49.8 \%$ of the $\mathrm{BH}$ of OA, LA, and ALA in EXP1, EXP2, and EXP3, respectively. Based on the model, BH of trans C18:1 FA was the rate-limiting step to complete BH. Importantly, oils were provided as triglycerides and the reported rates represent the rate of lipolysis and $\mathrm{BH}$. In conclusion, the rate of ruminal $\mathrm{BH}$ of $\mathrm{OA}, \mathrm{LA}$, and ALA was higher than that commonly observed in vitro, but the extent of $\mathrm{BH}$ was near expected values. The method developed provides a potential in vivo assay of ruminal $\mathrm{BH}$ for use in future experiments and modeling efforts.

Key words: biohydrogenation, unsaturated fatty acids, modeling, trans fatty acids

\section{Technical Note}

Unsaturated fatty acids (FA) are predominantly found in triglycerides in feeds and undergo biohydrogenation (BH) in the rumen, which enzymatically is lipolysis and a series of isomerization and hydrogenation steps. Metabolism of UFA by rumen microbes affects the profile of FA absorbed in the intestine and, subsequently, milk FA profile and physiology of the cow, especially milk fat yield (Bauman and Griinari, 2003). Understanding the rates and pathways of $\mathrm{BH}$ will allow development of improved nutritional models to maximize milk fat synthesis and modify milk FA profile. Microbial metabolism of UFA has been extensively studied in vitro using batch and continuous culture systems (Jenkins et al., 2008). These in vitro studies have provided essential insight into fundamental mechanisms and principles of $\mathrm{BH}$; however, specific aspects of in vitro systems may not reflect the microbial population 
and BH kinetics occurring within the rumen (Shingfield and Wallace, 2014). For example, most in vitro systems incubate fine-ground feed substrate with strained and buffer-diluted rumen fluid and are not expected to maintain viability of all microbial populations. Importantly, the microbial populations involved in $\mathrm{BH}$ have not been fully characterized, and it is not clear if all key populations are culturable, even in mixed cultures.

Duodenal and omasal digesta sampling and use of digesta flow markers has been considered the reference technique to assess the extent of $\mathrm{BH}$ (Fievez et al., 2007). However, this technique is costly and subject to considerable error associated with subsampling and marker bias (Owens and Hanson, 1992). Furthermore, this technique only reports the extent of $\mathrm{BH}$, unless coupled with rumen evacuation allowing calculation of FA BH and passage rate by the pool and flux method (Harvatine and Allen, 2006). The pool and flux approach limits mechanistic modeling of $\mathrm{BH}$ pathways and quantification of fast rates. Additionally, these intensive approaches make in vivo screening impractical. Modeling ruminal FA metabolism is also limited because ${ }^{13} \mathrm{C}$-labeled UFA are prohibitively expensive outside of in vitro culture. Our goal was to develop an alternative economical assay to assess in vivo rates, pathways, and extent of $\mathrm{BH}$. We hypothesized that rumen $\mathrm{BH}$ could be characterized using a perturbation trace model based on concentration pool of FA in the rumen.

Three 18-carbon UFA were characterized in separate experiments [EXP1: oleic acid (OA), EXP2: linoleic acid (LA), and EXP3: $\alpha$-linoleic (ALA)], each using 4 ruminally cannulated lactating Holstein cows. Cows were housed in a tiestall barn and all experimental procedures were approved by the Pennsylvania State University Institutional Animal Care and Use Committee. Cows received a contemporary lactating cow TMR composed of approximately (\% of DM) $40 \%$ corn silage, $15 \%$ alfalfa haylage, $3 \%$ grass hay, and $42 \%$ of a concentrate mix [ground corn, canola meal, cottonseed hulls, vitamin/mineral supplement, encapsulated urea (Optigen, Alltech Inc., Nicholasville, KY), bakery by-product meal, corn gluten meal, soybean hulls, and cane molasses]. The diet contained approximately (\% of DM) $17 \% \mathrm{CP}, 32 \% \mathrm{NDF}, 27 \%$ starch, and $3.1 \%$ total FA. Across the 3 experiments, DMI and milk yield averaged $25.3( \pm 1.5)$ and $38.5( \pm 6.8$, mean $\pm \mathrm{SD}) \mathrm{kg} / \mathrm{d}$, respectively.

The perturbation trace model included enrichment of rumen concentration pool of a targeted UFA and subsequent observation of disappearance of the UFA and formation of intermediate FA and stearic acid (Figure 1). Concentration pool enrichment was achieved by a single 200-g dose (i.e., bolus) of a UFA oil [predominantly triglyceride; EXP1: 87\% OA sunflower (Stratas Foods, Memphis, TN); EXP2: 70\% LA safflower (Jedwards International Inc., Braintree, MA); and EXP3: 54\% ALA flaxseed (Jedwards International Inc.)]. The UFA oil dose was based on the estimated rumen pool size and was evaluated in a pilot experiment in which $200 \mathrm{~g}$ was found adequate for enrichment of rumen UFA concentration pool without increasing total rumen FA to a level that may impair microbial activity. Under steady state conditions, either bolus or continuous infusion can be used to estimate kinetic rates of pools within a system (France and Dijkstra, 2005). A frequent feeding schedule was implemented with the goal of maintaining quasi-steady state ruminal conditions. Three days before the experiment, cows were fed in equal meals every $6 \mathrm{~h}$; the day before the experiment, cows were fed equal-sized meals every $2 \mathrm{~h}$; and on the day of the experiment, cows were fed hourly at $4.2 \% / \mathrm{h}(1 / 24 \mathrm{th})$ of expected daily DMI starting at $0730 \mathrm{~h}$. Refusals were removed only once per day.

An odd-chain SFA (C17:0 as free FA; Tokyo Chemical Industry Co., Tokyo, Japan) was included in the oil bolus in all experiments to serve as a marker of FA passage from the rumen. This requires the assumption that C17:0 passes at the same rate as the UFA under study. Stable isotope-labeled UFA would provide an ideal approach, but they are prohibitively expensive. Further validation of $\mathrm{FA}$ flow rates is warranted, but difficult to truly validate. Additionally, assuming that the ratio of C17:0 to the bolused unsaturated oil remains constant in the rumen, the odd-chain SFA also corrects for heterogeneity in sampling. In a pilot experiment using a similar single bolus approach, we observed that different odd-chain SFA (C11:0, C13:0, C15:0, and C17:0) disappeared from the rumen at different rates (Supplemental Figure S1; https://doi.org/jds.2017-13452). Briefly, C11:0 and C13:0 disappeared at unrealistically high rates (23.9 and $12.8 \% / \mathrm{h}$, respectively), considering that FA in the rumen are mainly bound to the surface of feed particles and, hence, expected to have passage rates similar to the solid phase (Jenkins et al., 2008). The fast disappearance of $\mathrm{C} 11: 0$ and $\mathrm{C} 13: 0$ could be related to a greater solubility of these FA in the liquid phase or their absorption across the rumen wall. Ruminal metabolism of C11:0 and C13:0 has not been investigated, to our knowledge, but early investigations have shown that the rumen epithelium was able to use even medium-chain SFA (ranging from C8:0 to C14:0) to produce ketone bodies (Noble, 1978). In contrast to the shorter-chain FA, C15:0 and C17:0 total disappearance rate (turnover) was 6.1 and $7.2 \% / \mathrm{h}$, respectively, which is similar to rates of FA passage described using the pool flux method (Harvatine and Allen, 2006). We selected C17:0 over C15:0, as it is found at a lower 
concentration in the rumen ( 0.4 vs. $0.8 \%$ of total FA), which facilitates concentration pool enrichment. Bolus infusion of $12 \mathrm{~g}$ of C17:0 routinely resulted in a $>5$-fold enrichment in the C17:0 concentration pool. The oil mixture (200 g of UFA oil $+12 \mathrm{~g}$ of C17:0) was placed in a water bath at $50^{\circ} \mathrm{C}$ for $30 \mathrm{~min}$ to aid mixing. Immediately before dosing at $1000 \mathrm{~h}$, approximately $20 \%$ of rumen contents were removed to provide space for initial mixing. The oil mixture was bolus-dosed through the rumen cannula and thoroughly mixed manually with the digesta for approximately $1 \mathrm{~min}$; then the removed digesta was returned and contents were thoroughly mixed for an additional $4 \mathrm{~min}$.

Rumen digesta was collected at $-1,-0.25,0.08,0.25$, $0.5,0.75,1,1.5,2,3,4$, and $6 \mathrm{~h}$ relative to the bolus. The first 2 samples $(-1$ and $-0.25 \mathrm{~h}$ ) were used to characterize background levels of each UFA in the rumen. The first sample after dosing was approximately $5 \mathrm{~min}$ relative to the bolus because of the time required for mixing. At each sampling, whole digesta was collected from 5 different locations in the rumen (cranial dorsal, cranial ventral, central, caudal dorsal, and caudal ventral), mixed in a bucket, and a composite subsample of approximately $200 \mathrm{~g}$ was collected and immediately placed on dry ice. A second subsample was strained through $0.5-\mathrm{mm}$ plastic mesh and $\mathrm{pH}$ was measured using a hand-held pH meter (model \# 35631-60, Oakton Instruments, Vernon Hills, IL); the remainder was immediately returned to the cow. Rumen samples were stored at $-20^{\circ} \mathrm{C}$ until lyophilized for $52 \mathrm{~h}$ with a 5 -step program $\left(-35^{\circ} \mathrm{C}\right.$ for $60 \mathrm{~min},-10^{\circ} \mathrm{C}$ for $120 \mathrm{~min}, 0^{\circ} \mathrm{C}$ for $480 \mathrm{~min}, 3^{\circ} \mathrm{C}$ for $1,250 \mathrm{~min}, 30^{\circ} \mathrm{C}$ for $1,250 \mathrm{~min}$; Virtis 3.5 L XL, The Virtis Co., Gardiner, NY). Digesta was then ground to $<1 \mathrm{~mm}$ using a spinning blade coffee grinder (Hamilton Beach, model 80335R). Fatty acids were methylated using sodium methoxide followed by methanolic $\mathrm{HCl}$, as described by Jenkins (2010). Internal standards for determination of FA concentrations were cis-10 C17:1 free FA (also verified methylation efficiency) and C19:0 FAME.

Fatty acid methyl esters were quantified using a GC (Agilent 6890; Agilent Technologies, Palo Alto, CA) equipped with a fused-silica capillary column (SP-2560; $100 \mathrm{~m} \times 0.25 \mathrm{~mm}$ i.d. with $0.2-\mu \mathrm{m}$ film thickness; Supelco Inc., Bellefonte, PA) and a flame-ionization detector. Constant carrier gas flow was $1 \mathrm{~mL} / \mathrm{min}$ of

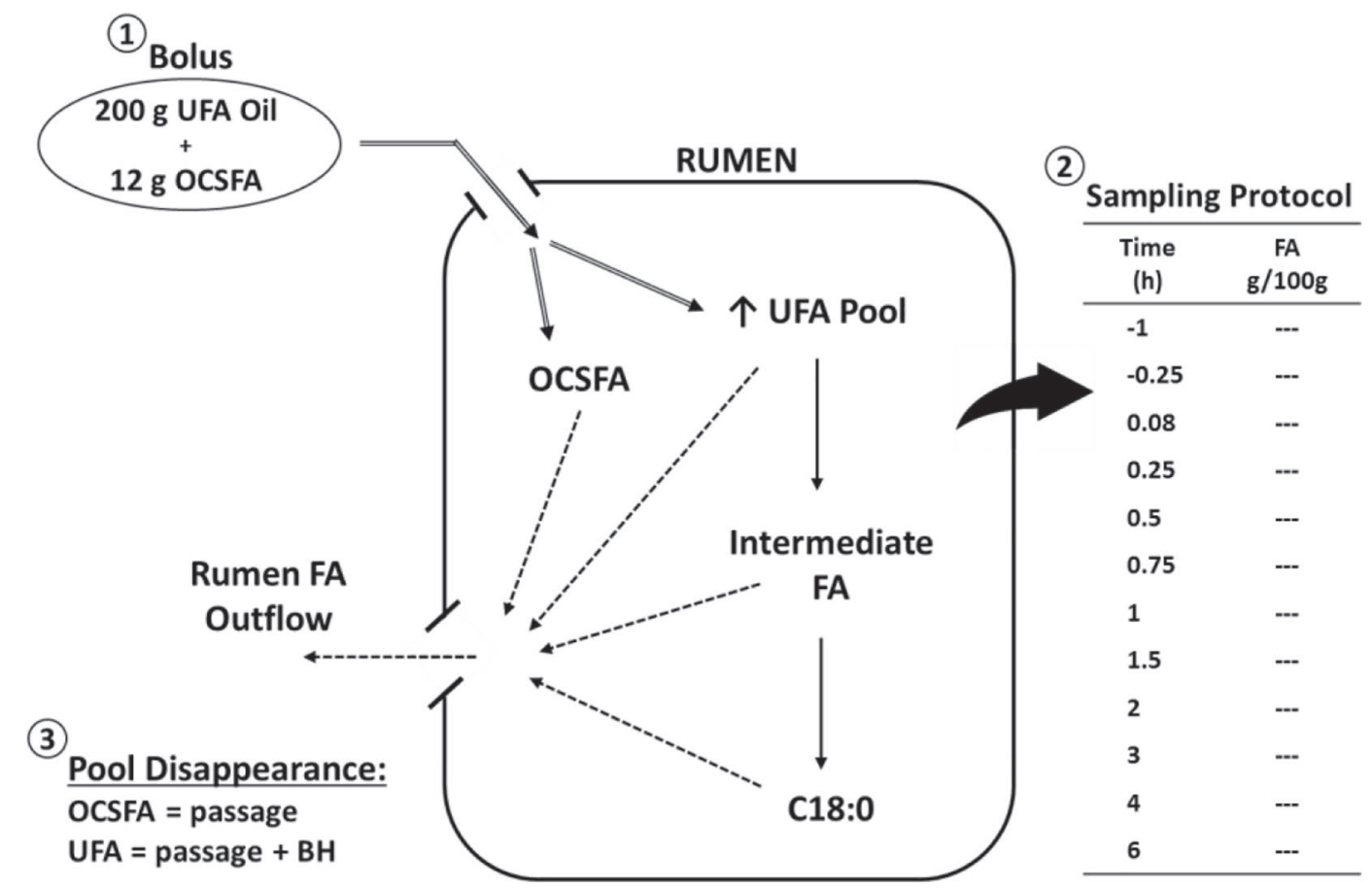

Figure 1. Schematic design of the perturbation trace model used to study rumen biohydrogenation (BH) in vivo. (1) Perturbation of a concentration pool (enrichment) is achieved by a single dose infusion (i.e., bolus) of a UFA oil rich in the UFA being studied. The bolus is manually mixed with rumen contents through the rumen cannula. (2) Samples of whole rumen contents are collected over time and fatty acid (FA) profile is determined by GC. Samples before bolus establish the background levels, whereas the remaining samples describe the disappearance of the UFA being studied and formation of intermediates. (3) The rate of BH of an UFA can be calculated from its total disappearance (i.e., turnover rate) assuming that (a) the odd-chain SFA (OCSFA) disappears only by passage, (b) the UFA disappears by passage and BH, and (c) the rate of passage of the OCSFA and UFA are equal. Double-lined arrows represent input, solid arrows represent BH, and dashed arrows the passage of FA out of the rumen. 
hydrogen and detector gas flows were $25 \mathrm{~mL} / \mathrm{min}$ of hydrogen, $300 \mathrm{~mL} / \mathrm{min}$ of air, and $40 \mathrm{~mL} / \mathrm{min}$ of nitrogen plus carrier. The injection volume was $1 \mu \mathrm{L}$ of FAME in hexane, with a 100:1 split ratio. Initial oven temperature was $45^{\circ} \mathrm{C}$ for $4 \mathrm{~min}$, after which it was increased $13^{\circ} \mathrm{C} /$ min to $150^{\circ} \mathrm{C}$ and held for $47 \mathrm{~min}$, and then increased $4^{\circ} \mathrm{C} / \mathrm{min}$ to $215^{\circ} \mathrm{C}$ and held for $25 \mathrm{~min}$. Peaks were identified using reference standards (GLC 780, 461, and 566, NuChek Prep Inc., Elysian, MN; Bacterial Acid Methyl Ester Mix, 47080-U, Sigma-Aldrich Inc., St. Louis, MO; and GLC 110 mixture, Matreya LLC., State College, PA). Recoveries of individual FA were determined using an equal weight reference standard (GLC 461; NuChek Prep Inc.).

Data were modeled to characterize the rate of metabolism and was conducted using the geometric mean at each time point (4th root of the product of means), unless otherwise indicated. The use of geometric mean is appropriate for nonlinear models working with concentrations, as it better represents the relationship between the variance of concentration and its mean (Lindsey, 2001; Lopez-Teros et al., 2017). The objective of the current experiment was not to make statistical comparisons between experiments or treatments. If the assay is implemented in such an experiment, $\mathrm{BH}$ should be modeled within cow or in a mixed model to allow statistical inference on model parameters. The standard deviation, fractional standard deviation, or standard error of the mean was used to evaluate variation in the data or uncertainty of fitted models.

The hourly feeding schedule was implemented on the day of the bolus to stabilize intake and establish near steady state rumen conditions, although a non-bolus control was not included. Rumen $\mathrm{pH}$ remained stable and averaged $6.7 \pm 0.12,6.6 \pm 0.11$, and $6.7 \pm 0.09$ (mean $\pm \mathrm{SD}$ ) in the OA, LA, and ALA experiments, respectively (Supplemental Figure S2; https://doi.org/ $10.3168 /$ jds.2017-13452). The variation in rumen $\mathrm{pH}$ was much smaller than the fluctuation across the day commonly observed in cows consuming TMR. Rumen concentrations of C15:0 and C24:0 were used to assess background kinetics within each experiment, as these 2 FA were not present in the bolus oil. Throughout the assay, C15:0 averaged $0.56 \pm 0.06 \mathrm{~g} / 100 \mathrm{~g}$ of FA and C24:0 averaged $0.47 \pm 0.03 \mathrm{~g} / 100 \mathrm{~g}$ of FA across the 3 experiments (data not shown). The 10.2 and $6.9 \%$ variation around the mean for $\mathrm{C} 15: 0$ and $\mathrm{C} 24: 0$, respectively, indicate that background kinetics were maintained close to steady state.

The 200-g triglyceride bolus successfully enriched the concentration of the UFA under investigation. Specifically, as a percent of FA, OA increased from 8.9 to $30.1 \%$ of FA in EXP1, LA was increased from 11.1 to $35.9 \%$ of $\mathrm{FA}$ in EXP2, and ALA was increased from
2.1 to $19.8 \%$ of FA in EXP3 (Figure 2A). As a percent of DM, OA was increased from 0.5 to $2.7 \%$ of DM in EXP1, LA was increased from 0.5 to $2.4 \%$ in EXP2, and ALA was increased from 0.1 to $1.3 \%$ in EXP 3 (Figure 2B). Importantly, the bolus increased the total amount of FA in digesta to $7.3 \pm 1.4 \%$ of DM (Table 1 ), which is below that expected to majorly impair microbial activity and disrupt fermentation (Jenkins, 1993). Under different scenarios, this 200-g dose may need to be adjusted slightly to accommodate differences in fat concentration in the basal diet, rumen pool size, and DMI, among other factors. As expected, OA and LA were 8.9 and $11.1 \%$ of total rumen FA before the bolus, respectively, and much higher than ALA (2.1\%). The higher background of OA and LA limited the enrichment that could be achieved (Figure 2). A stable isotope approach would allow the use of a very small bolus that would not appreciably increase FA concentration and would have fewer concerns related to possible enzyme saturation, as discussed below; however, enriched ${ }^{13} \mathrm{C}$ UFA are prohibitively expensive.

The UFA of interest reached prebolus concentration within $4 \mathrm{~h}$ in all experiments (Figure 2). A small increase in the concentration of OA and LA at the last sampling was noted ( $6 \mathrm{~h}$ postbolus), possibly due to increased feed intake, as both OA and LA were higher in the diet. Refusals were not removed at each feeding and cows that had not consumed all their feed during earlier feedings may have increased intake at this time, which would have increased rumen OA and LA. As the UFA of interest reached baseline before this, the $6 \mathrm{~h}$ time point was excluded with no effect on characterization of rates. It is recommended that, in future experiments, researchers remove refused feed at each feeding to maintain tighter controls on intake during the procedure. A slightly lower feeding rate would also provide less refusals or refusals could be put into the rumen through the cannula; however, care should be taken, as severe restriction may cause a decrease in rumen pool size, and particle size reduction may be needed before feed is placed through the cannula. Importantly, near steady state conditions appear to have been maintained during the first $4 \mathrm{~h}$, as discussed above.

The return of enriched UFA to baseline concentration (both passage and $\mathrm{BH}$ ) was modeled using the firstorder kinetic model of Ørskov and McDonald (1979), implemented in the nonlinear procedure of JMP Pro 12.1 (SAS Institute Inc., Cary, NC). The model was Y $=\mathrm{C}+\mathrm{Pe}^{(-\mathrm{k} \times \mathrm{t})}+\varepsilon$, where $\mathrm{Y}=$ concentration of UFA $(\mathrm{g} / 100 \mathrm{~g}$ of $\mathrm{FA})$ at time $\mathrm{t}$; $\mathrm{C}=$ unavailable UFA; $\mathrm{Pe}=$ available UFA; $\mathrm{k}=$ fractional rate of UFA disappearance $\left(\mathrm{h}^{-1}\right) ; \mathrm{t}=$ time $(\mathrm{h})$; and $\varepsilon=$ residual error. Firstorder kinetics were previously used to describe ruminal metabolism of UFA under different systems (Beam et 


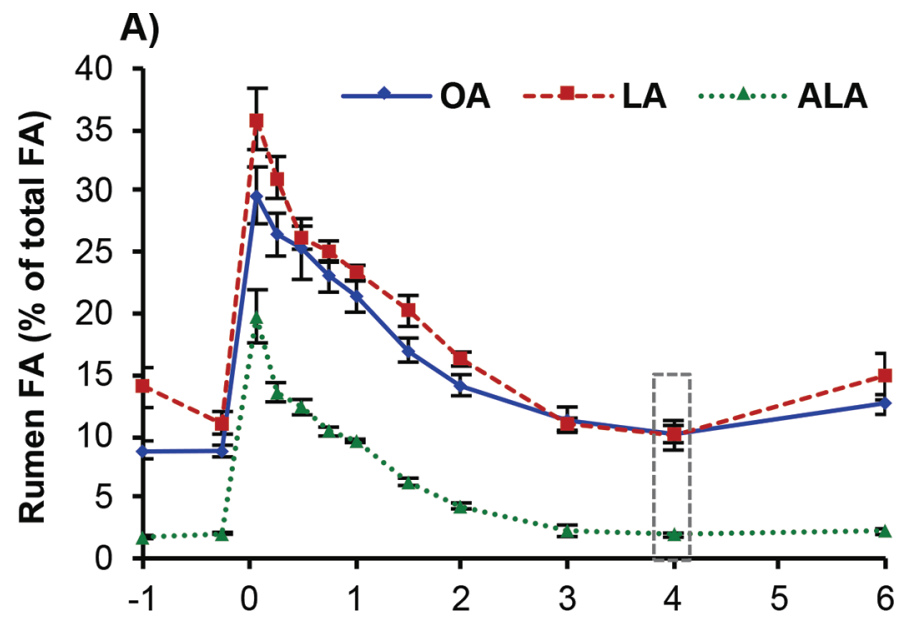

B)

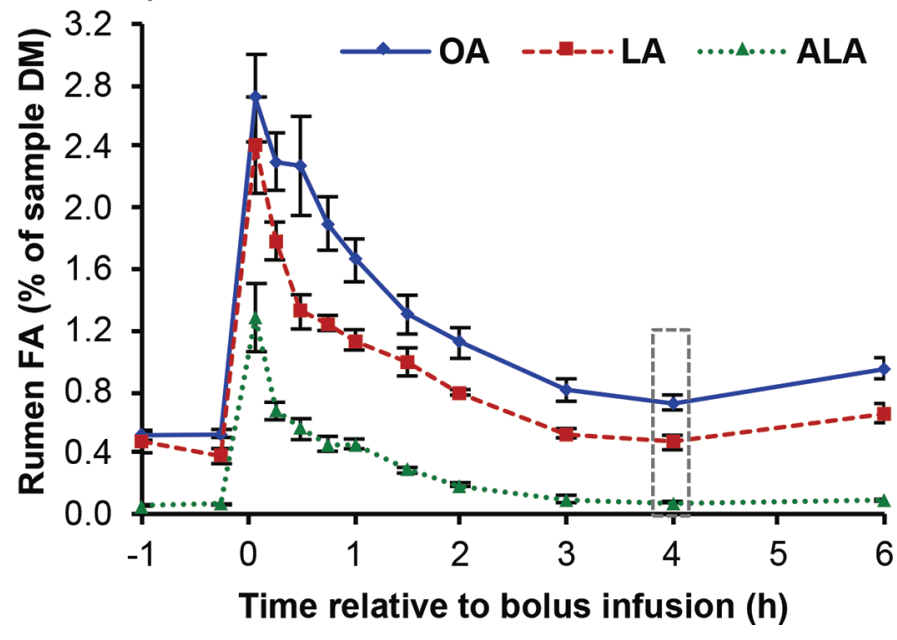

Figure 2. Perturbation of rumen concentration of oleic (OA), linoleic (LA), and $\alpha$-linolenic acid (ALA) as a percentage of total fatty acids (A) and percentage of DM (B) in experiments 1,2 , and 3 , respectively. Enrichment of the UFA is achieved by a bolus infusion of $200 \mathrm{~g}$ of an oil enriched in the fatty acid (FA). Data represent the geometric mean (error bars $=$ SEM) of 4 cows within each independent experiment. The enriched FA reached prebolus levels by $4 \mathrm{~h}$ after bolus, as shown by the dashed rectangle. Color version available online. al., 2000; Harvatine and Allen, 2006; Ribeiro et al., 2007). We used the approach described by Ribeiro et al. (2007) to test if first-order kinetics applied to OA, LA, and ALA in the current experiments. We found that the natural logarithm of FA concentration versus time resulted in a linear plot (first order, $P<0.001$ for all), the inverse of FA concentration versus time was not linear (i.e., not second order), and the residuals versus predicted for the natural logarithm of FA concentration versus time did not make any discernable pattern. However, Moate et al. (2008) reported that BH of LA fit Michaelis-Menten kinetics in vitro.

A discrete time lag was tested, but was not different from zero for all 3 UFA (data not shown). This was expected, as the triglycerides present in the bolus oil are readily available to microbes and hydrolysis is expected to be rapid (Harfoot and Hazlewood, 1997; Beam et al., 2000). Few experiments separate the rates of hydrolysis and $\mathrm{BH}$, and lipolysis may be inhibited in some situations including increasing trilinolein and low pH (Jenkins et al., 2008). Moate et al. (2008) determined that hydrolysis of the first FA from trilinolein fit Michaelis-Menten kinetics, but that the subsequent steps followed linear rates. In the current experiment, data were modeled starting $5 \mathrm{~min}$ after bolus dosing, because of the time required for mixing, and FA were not presented in pure form as investigated by Moate et al. (2008). Additionally, in vitro batch culture is expected to have a greater lag as microbes adapt to the system and substrate become available compared with the in vivo approach used in the present study. Of note, an unavailable concentration pool (C fraction) did not parameterize in the disappearance of $\mathrm{C} 17: 0$, indicating that $\mathrm{C} 17: 0$ background was constant and C17:0 disappearance was of bolus origin only. Additionally, to minimize variation due to mixing, we did not include the first time point after the bolus in analysis of C17:0.

The fractional rate of ruminal disappearance of $\mathrm{OA}$ was $54.1 \% / \mathrm{h}$, LA was $60.5 \% / \mathrm{h}$, and ALA was $93.0 \% / \mathrm{h}$

Table 1. Increase in concentration of oleic (OA), linoleic (LA), and $\alpha$-linolenic (ALA) acid in rumen digesta immediately following the bolus infusion ${ }^{1}$

\begin{tabular}{|c|c|c|c|c|c|}
\hline \multirow[b]{2}{*}{ Item } & \multirow{2}{*}{$\frac{\% \text { Rumen DM }}{\text { Total }_{\mathrm{FA}^{2}}}$} & \multicolumn{4}{|c|}{$\%$ of Total FA $($ mean \pm SD $)$} \\
\hline & & $\mathrm{C} 17: 0$ & $\mathrm{OA}$ & LA & ALA \\
\hline Prebolus & $3.9 \pm 1.4$ & $0.4 \pm 0.1$ & $8.9 \pm 1.0$ & $11.1 \pm 1.8$ & $2.1 \pm 0.1$ \\
\hline Postbolus $^{3}$ & $7.3 \pm 1.4$ & $2.5 \pm 0.5$ & $30.1 \pm 4.6$ & $35.9 \pm 5.0$ & $19.8 \pm 4.3$ \\
\hline Fold-enrichment ${ }^{4}$ & $0.8 \pm 0.4$ & $5.1 \pm 0.9$ & $2.4 \pm 0.4$ & $2.2 \pm 0.2$ & $8.6 \pm 1.8$ \\
\hline
\end{tabular}

${ }^{1}$ Enrichment of total fatty acids (FA) and C17:0 pools were calculated within experiment and reported values represent an average across the 3 experiments. Enrichment of OA, LA, and ALA represent the geometric mean of 4 cows within each independent experiment.

${ }^{2}$ Total FA $(\% \mathrm{DM})$ before the bolus averaged $4.9( \pm 0.48), 3.42( \pm 0.33)$, and $3.06( \pm 0.36)$ for OA, LA, and ALA, respectively.

${ }^{3}$ Concentration of the first sample collected immediately after the bolus $(\sim 5 \mathrm{~min})$.

${ }^{4}$ Calculated as (Pre + Post) $/$ Post. 
in EXP1, EXP2, and EXP3, respectively, modeled based on percent of FA data. This rate includes both hydrolysis and initial isomerization, as the UFA was in a triglyceride. The data were also modeled based on percent of DM and resulted in similar values when excluding the time zero value to ensure adequate mixing (data not shown). The difference between using the geometric mean of all data points was generally $<3.5 \%$ (data not shown). The fractional rate of C17:0 disappearance was 9.1, 6.9, and 5.2\%/h in EXP1, EXP2, and EXP3, respectively. These differences may be due to differences in DMI or forage digestion rates between the experiments. It is worth noting that passage rate was low compared with turnover rate, and thus has a smaller effect. We defined the disappearance of UFA due to $\mathrm{BH}\left(\mathrm{k}_{\mathrm{bh}}\right)$ and then calculated its fractional rate as the difference between total disappearance rate (turnover; $\mathrm{k}_{\mathrm{bh}}+\mathrm{k}_{\mathrm{p}}$ ) and passage rate $\left(\mathrm{k}_{\mathrm{p}}\right)$. This required the assumptions (a) C17:0 disappeared only by passage, (b) UFA disappeared by passage and $\mathrm{BH}$, (c) steady state microbial synthesis of C17:0, (d) equal microbial uptake of C17:0 and UFA, and (e) equal passage rate of $\mathrm{C} 17: 0$ and UFA. Under this model, the rate of hydrolysis and initial isomerization of OA was $45.0 \% / \mathrm{h}, \mathrm{LA}$ was $53.6 \% / \mathrm{h}$, and ALA was $87.8 \% / \mathrm{h}$ in EXP1, EXP2, and EXP3, respectively (Figure 3D). Lipolysis may vary due to amount and source of lipid used (Beam et al., 2000). Lipolysis was not expected to be rate limiting in the current experiment based on previous work (Harfoot and Hazlewood, 1997; Jenkins et al., 2008), but should be tested in future experiments by comparison of a triglyceride and free FA. If lipolysis was rate limiting, this means the actual rate of isomerization was faster than the reported rates. Using the $\mathrm{k}_{\mathrm{bh}} /\left(\mathrm{k}_{\mathrm{bh}}+\mathrm{k}_{\mathrm{p}}\right)$ principle, we calculated that the extent of $\mathrm{BH}$ was 85.6, 89.8, and $94.7 \%$ for OA, LA, and ALA in their respective experiments.

The in vivo method developed resulted in the expected extent of $\mathrm{BH}$, but the rates of ruminal $\mathrm{BH}$ were 2 to 3 times higher, depending on the UFA, than those observed in vitro by others (Beam et al., 2000; Ribeiro et al., 2007). One concern with a perturbation model was saturation of enzyme capacity. Because observed rates were high and background levels were achieved within $4 \mathrm{~h}$, it does not appear that $\mathrm{BH}$ capacity was overrun. We would have expected lower rates than previously reported if saturation had occurred. Thus, results obtained with this method could represent the maximal rates of $\mathrm{BH}$ under normal ruminal fermentation. Importantly, ruminal metabolism of UFA in feeds is expected to be lower because release of FA from the feed matrix may be rate limiting.

The method allowed observation of formation of intermediates of the bolused UFA (Figure 4). After
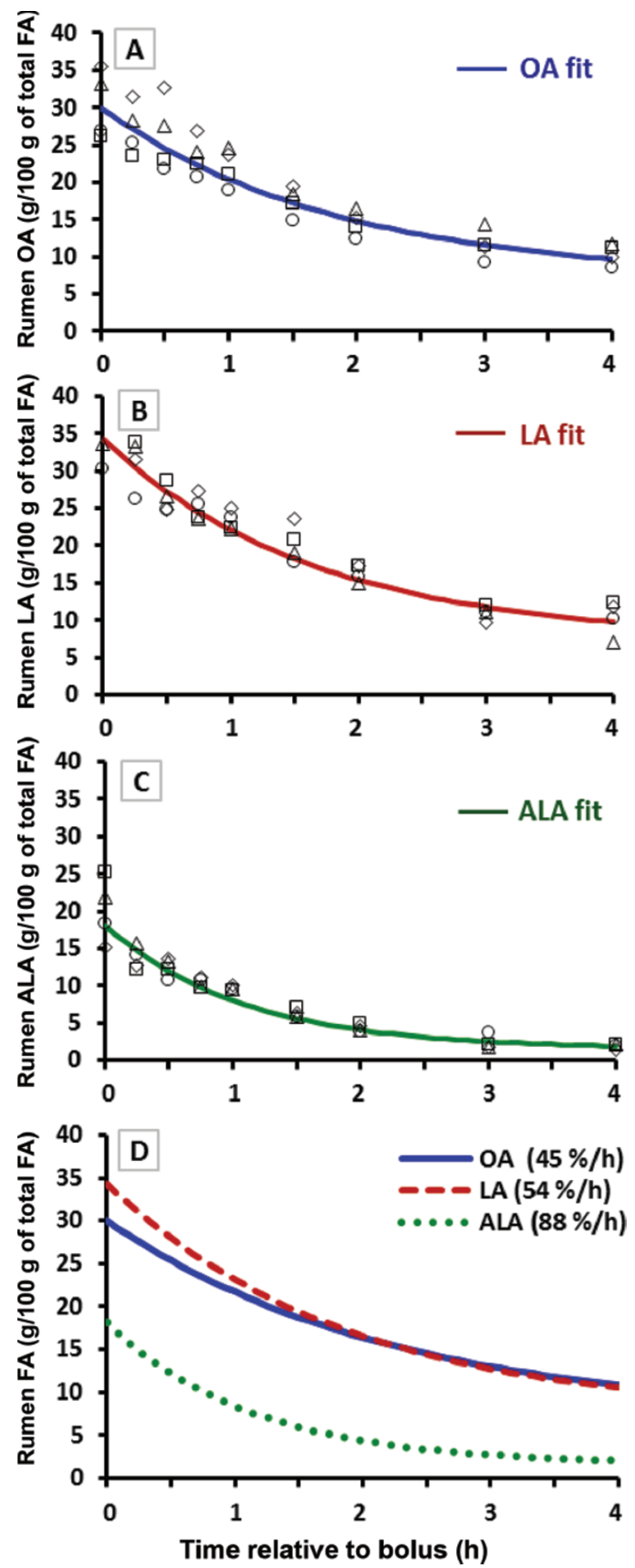

Figure 3. Ruminal disappearance of oleic (OA, A), linoleic (LA, $\mathrm{B}$ ), and $\alpha$-linolenic acid (ALA, C) following enrichment by a bolus. Lines are the fit to a single available pool, first-order kinetic decay described by the model: $\mathrm{Y}=\mathrm{C}+\mathrm{Pe}^{(-\mathrm{k} \times \mathrm{t})}+\varepsilon$, where $\mathrm{Y}=$ concentration of UFA ( $\mathrm{g} / 100 \mathrm{~g}$ of fatty acids) at time t, $\mathrm{C}=$ unavailable UFA, Pe $=$ available UFA, $\mathrm{k}=$ fractional rate of UFA disappearance $\left(\mathrm{h}^{-1}\right) ; \mathrm{t}=$ time $(\mathrm{h})$, and $\varepsilon=$ residual error. Model was fit to the geometric mean of 4 cows within each independent experiment. Individual cow data are shown with different markers for each cow. Root mean squared errors were $0.74,1.34$, and 1.21 for the model of OA, LA, and ALA, respectively. Observed data for cow $1(\square)$, cow $2(\diamond)$, cow $3(\Delta)$, and cow $4(\bigcirc)$ are also plotted. Panel D depicts model-predicted biohydrogenation rates $(\% / \mathrm{h})$ of OA, LA, and ALA following enrichment by a bolus. In this model, biohydrogenation equals total rate of UFA disappearance minus rate of fatty acid (FA) passage based on C17:0 disappearance. Color version available online. 
the OA bolus, we noted an increase in trans-10 C18:1 predominantly, but also a smaller increase in trans-11 C18:1 and other trans C18:1 isomers. Following the LA bolus was an increase in cis-9,trans-11 CLA predominantly, but also a smaller increase in trans-10, cis-12 CLA. The increase in the CLA isomers was followed by an increase in trans-10 C18:1 and trans-11 C18:1. After blousing ALA, we observed an increase in cis-9, trans11,cis-15 C18:3, which was followed by a large increase in trans-11,cis-15 C18:2 and trans-11 C18:1.

A key goal of our work was to develop a method that quantified the formation and metabolism of intermediates of ruminal BH. Ribeiro et al. (2007) previously modeled metabolism of UFA in batch culture using a compartmental model. We used previously established $\mathrm{BH}$ pathways and time course data for similar compartmental modeling (Supplemental Figures S3, S4, and S5; https://doi.org/10.3168/jds.2017-13452). The compartmental model included both the predominant (normal) $\mathrm{BH}$ pathways of OA, LA, and ALA and the alternate BH pathway (trans-10 shift) that occurs during $\mathrm{BH}$-induced milk fat depression, as described by Griinari and Bauman (1999). The disappearance of the UFA (OA, LA, and ALA) that was not accounted for by these $2 \mathrm{BH}$ pathways was assigned to a concentration pool of other isomers. The normal $\mathrm{BH}$ pathways modeled in our study included OA reduced directly to stearic acid; LA initially isomerized to cis-9,trans-11 18:2 followed by reduction to trans-11 18:1; and ALA initial isomerization producing cis-9,trans-11,cis-15 18:3, followed by reduction to trans-11,cis-15 18:2 and reduction to trans-11 18:1. The alternate $\mathrm{BH}$ pathways modeled included OA isomerized to either trans 6-8, trans-9, trans-10, trans-11, or trans-12 C18:1 and LA initial isomerization producing trans-10,cis-12 18:2, followed by a reduction to trans-10 18:1. The alternate pathway of ALA BH (initial isomerization producing trans-10,cis-12,cis-15 18:3, followed by reduction to trans-10,cis-15 18:2 and reduction to trans-10 18:1) was not modeled because the trans isomers in this pathway could not be identified under the GC conditions used. Therefore, it is possible that the trans-11,cis-15 18:2 concentration pool might have been inflated if coelution with trans-10,cis-15 18:2 occurred.

Compartmental modeling was carried out using WinSAAM (www.winsaam.org), the Windows version of the Simulation, Analysis and Modeling software (Wastney et al., 1999). The concentration of the 3 UFA and their intermediates during $\mathrm{BH}$ from 0 to $4 \mathrm{~h}$ was used to estimate the fractional transfer rates among concentration pools. The geometric mean for the 4 cows within each experiment was weighted using the fractional standard deviation $(\mathrm{FSD}=$ mean/standard deviation). In this model, each FA represented a compartment; however,
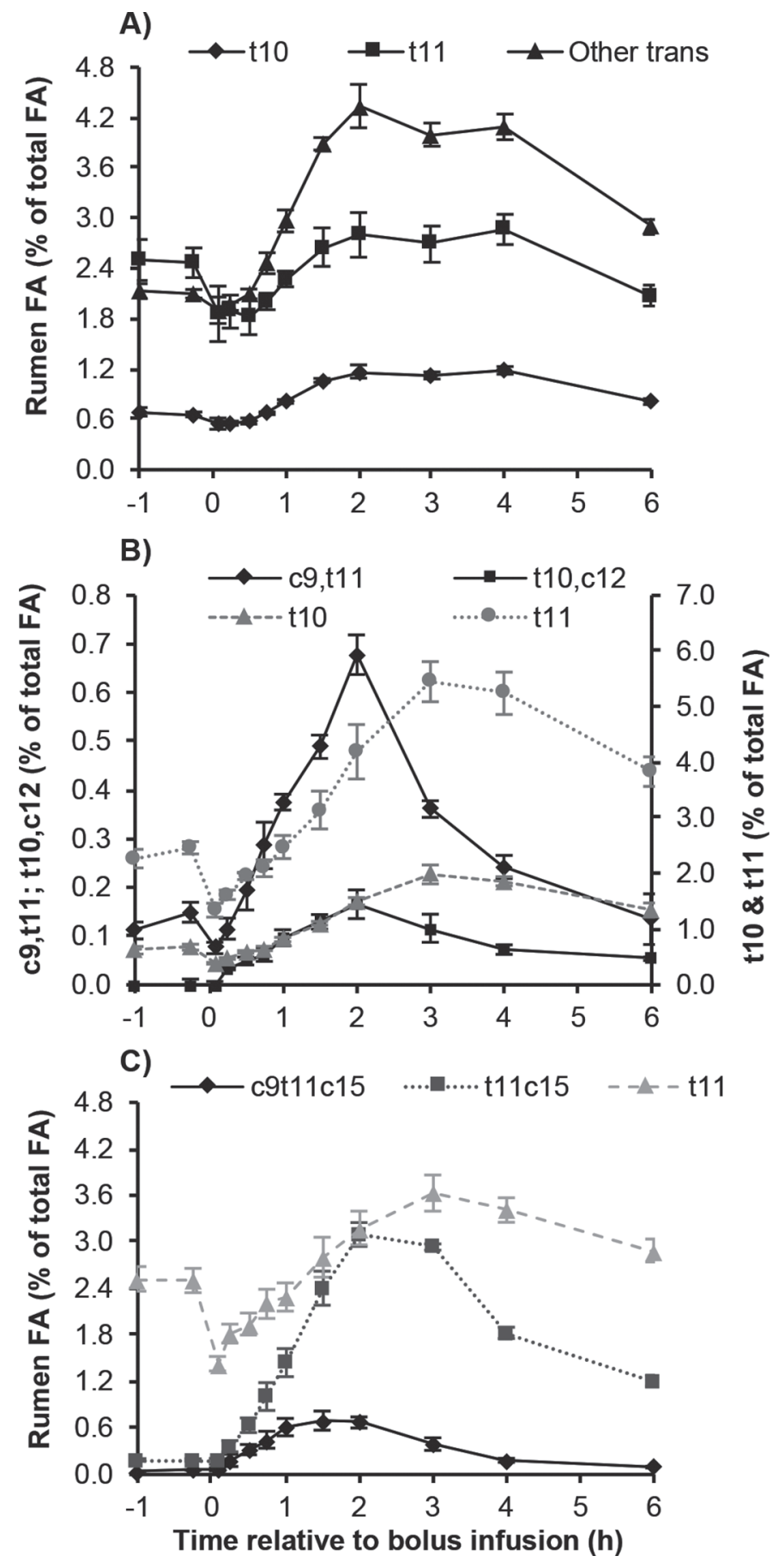

Figure 4. Ruminal appearance of intermediates of oleic (OA, A), linoleic (LA, B), and $\alpha$-linolenic acid (ALA, C) metabolism following enrichment by a bolus in experiments 1,2 , and 3 , respectively. Enrichment of the UFA is achieved by a bolus infusion of $200 \mathrm{~g}$ of an oil enriched in the fatty acid (FA). Data represent the geometric mean (error bar $=\mathrm{SEM})$ of 4 cows within each independent experiment. $\mathrm{t}$ $=$ trans; $\mathrm{c}=$ cis.

some UFA required the subdivision of the compartment into 2 concentration pools, one that described changes related to the bolus and another characterizing basal 
levels (a constant pool that has no dynamic elements consistent with steady state conditions). The compartmental models were developed independently in a stepwise manner for each UFA. First, the disappearance of C17:0 was modeled and this rate was subtracted from the total disappearance rate of each compartment to account for passage of FA from the rumen. Next, the disappearance of OA, LA, or ALA was modeled as a single exponential decay. Subsequently, FA intermediates (compartments) were added and modeled one at a time until the pathway reached stearic acid or could not be further modeled. The starting parameter estimates for direct transfers were based on published data (see review by Shingfield and Wallace, 2014), and the addition of a transfer rate was made only if it improved model fit and decreased the residual sum of squares. Manual adjustments of the parameter values were made first and then the gradient optimizer function of WinSAAM was applied. This command performs a series of iterations that attempt to converge the model to the lowest residual sum of squares possible. The parameters are presented as fractional transfer rates (or the fraction of FA transferred to compartment I from compartment J per unit of time), and the statistical uncertainty as fractional standard deviation (Supplemental Figures S3, S4, and S5; https://doi.org/10.3168/jds .2017-13452). Fractional transfer rates are nonlinearly estimated, are time-invariant, and represent the percent transfer of FA between 2 compartments per unit of time (instantaneous rate of change).

The rates of rumen disappearance and $\mathrm{BH}$ estimated by the compartmental model were similar to those estimated by the single available pool, first-order kinetic approach (Figure 3 and Supplemental Figures S3, S4, and S5; https://doi.org/10.3168/jds.2017-13452). In the compartmental analysis, 2 transfer rates were required to model the isomerization of $\mathrm{OA}$ to alternate trans-18:1 isomers, LA to either cis-9,trans-11 or trans10,cis-12 18:2, and ALA to cis-9,trans-11,cis-15 18:3 (Supplemental Figures S3, S4, and S5, respectively). Modeling the flux as 2 different rates considerably decreased the sum of squares in all the above-mentioned compartments. The second transfer took place approximately 35 min after the bolus infusion (Supplemental Figure S6; https://doi.org/10.3168/jds.2017-13452), but was much faster than the first and was a larger contributor to isomerization. This result is compatible with the fact that multiple bacterial species, with different work capacities, are thought to be involved in ruminal isomerization and BH of UFA (Shingfield and Wallace, 2014). Two direct transfers from LA to trans-11 18:1 and trans-10 18:1 and from ALA to trans11,cis-15 were also required to model these intermediate compartments (Supplemental Figures S4 and S5, respectively). As in the case of the first intermediate, one rate contained a time delay (Supplemental Figure S7; https://doi.org/10.3168/jds.2017-13452) but accounted for the majority of the FA flux through that pathway. Direct BH without accounting for isomerization has been previously modeled by Harvatine and Allen (2006) and may indicate that these BH steps are performed by a group of bacteria that possesses enzymatic machinery to isomerize and hydrogenate double bonds quickly. However, this hypothesis could not be tested within the current experimental procedures.

The compartmental model allowed calculation of turnover time in addition to transfer rates. We observed that the turnover time for isomerization and reduction of the first double bond of ALA and LA occurred within 26 and $48 \mathrm{~min}$, respectively (Supplemental Table S1; https://doi.org/10.3168/jds.2017-13452); in contrast, hydrogenation of trans-C18:1 FA occurred less rapidly (181 min, Supplemental Table S1). These results are in agreement with previous in vitro work (Singh and Clement Hawke, 1979) and indicate that hydrogenation of trans-C18:1 FA is a rate-limiting step in the complete BH of UFA, which consequently increases rumen passage of the trans intermediates. Lastly, our method was effective in quantifying the amount of each UFA that was metabolized through normal or alternative BH pathways. The normal pathway explained 49.8, 37.3 , and $46.0 \%$ of BH of ALA, LA, OA, respectively (Supplemental Table S1).

In conclusion, the in vivo perturbation method resulted in the expected $\mathrm{BH}$ intermediates and extent of $\mathrm{BH}$; however, the rates of $\mathrm{BH}$ were much greater than those commonly observed in vitro. The method developed provides a potential economical in vivo assay of ruminal $\mathrm{BH}$ for use in future experiments to quantify the effects of dietary factors on rumen $\mathrm{BH}$ rates and pathways. Additional improvement would be obtained if the method were coupled with more advanced techniques of FA analysis (e.g., GC-MS), which would increase the number of individual intermediate FA pools that could be incorporated into the compartmental analysis.

\section{ACKNOWLEDGMENTS}

The authors thank the staff of the Penn State Dairy Farm for their conscientious care of the experimental cows. The help of Jocely de Souza, Jackie Ying, Yifan Fan, Rebecca Bomberger, and Isaac Salfer with sample collection and analysis is greatly appreciated (all from Penn State University). We also acknowledge modeling assistance and discussion from Natalie Urrutia and Jennifer Ford (Penn State University) and technical editing from Joanne Green (Penn State University). 


\section{REFERENCES}

Bauman, D. E., and J. M. Griinari. 2003. Nutritional regulation of milk fat synthesis. Annu. Rev. Nutr. 23:203-227.

Beam, T. M., T. Jenkins, P. Moate, R. Kohn, and D. Palmquist. 2000. Effects of amount and source of fat on the rates of lipolysis and biohydrogenation of fatty acids in ruminal contents. J. Dairy Sci. 83:2564-2573

Fievez, V., B. Vlaeminck, T. Jenkins, F. Enjalbert, and M. Doreau. 2007. Assessing rumen biohydrogenation and its manipulation in vivo, in vitro and in situ. Eur. J. Lipid Sci. Technol. 109:740-756.

France, J., and J. Dijkstra. 2005. Volatile fatty acid production. Pages 157-175 in Quantitative Aspects of Ruminant Digestion and Metabolism. 2nd ed. J. Dijkstra, J. M. Forbes, and J. France, ed. CAB International, Wallingford, UK.

Griinari, J., and D. E. Bauman. 1999. Biosynthesis of conjugated linoleic acid and its incorporation into meat and milk in ruminants. Pages 180-200 in Advances in Conjugated Linoleic Acid Research. vol. 1. American Oil Chemists' Society, Champaign, IL.

Harfoot, C., and G. Hazlewood. 1997. Lipid metabolism in the rumen. Pages 382-426 in The Rumen Microbial Ecosystem. 2nd ed. P. N. Hobson, C. S. Stewart, ed. Blackie Academic and Professional, London, UK.

Harvatine, K. J., and M. S. Allen. 2006. Fat supplements affect fractional rates of ruminal fatty acid biohydrogenation and passage in dairy cows. J. Nutr. 136:677-685.

Jenkins, T. C. 2010. Technical note: Common analytical errors yielding inaccurate results during analysis of fatty acids in feed and digesta samples. J. Dairy Sci. 93:1170-1174.

Jenkins, T. C., R. Wallace, P. Moate, and E. Mosley. 2008. Boardinvited review: Recent advances in biohydrogenation of unsaturated fatty acids within the rumen microbial ecosystem. J. Anim. Sci. 86:397-412.

Jenkins, T. C. 1993. Lipid metabolism in the rumen. J. Dairy Sci. 76:3851-3863.
Lindsey, J. K. 2001. Nonlinear Models in Medical Statistics. Oxford University Press, Oxford, UK.

Lopez-Teros, V., J. L. Ford, M. H. Green, G. Tang, M. A. Grusak, L. Quihui-Cota, T. Muzhingi, M. Paz-Cassini, and H. AstiazaranGarcia. 2017. Use of a "super-child" approach to assess the vitamin A equivalence of Moringa oleifera leaves, develop a compartmental model for vitamin A kinetics, and estimate vitamin A total body stores in young Mexican children. J. Nutr. 147:2356-2363. https:// doi.org/10.3945/jn.117.256974.

Moate, P. J., R. C. Boston, T. C. Jenkins, and I. J. Lean. 2008. Kinetics of ruminal lipolysis and triacylglycerol and biohydrogenation of long-chain fatty acids: new insights from old data. J. Dairy Sci. 91:731-742.

Noble, R. C. 1978. Digestion, absorption and transport of lipids in ruminant animals. Prog. Lipid Res. 17:55-91.

Ørskov, E., and I. McDonald. 1979. The estimation of protein degradability in the rumen from incubation measurements weighted according to rate of passage. J. Agric. Sci. 92:499-503.

Owens, F. N., and C. F. Hanson. 1992. External and internal markers for appraising site and extent of digestion in ruminants. J. Dairy Sci. 75:2605-2617.

Ribeiro, C. V., M. Eastridge, J. Firkins, N. St-Pierre, and D. Palmquist. 2007. Kinetics of fatty acid biohydrogenation in vitro. J. Dairy Sci. 90:1405-1416.

Shingfield, K., and R. Wallace. 2014. Synthesis of Conjugated Linoleic Acid in Ruminants and Humans. Royal Society of Chemistry, London, UK.

Singh, S., and J. Clement Hawke. 1979. The in vitro lipolysis and biohydrogenation of monogalactosyldiglyceride by whole rumen contents and its fractions. J. Sci. Food Agric. 30:603-612.

Wastney, M. E., B. H. Patterson, O. A. Linares, P. C. Greif, and R. C. Boston. 1999. Investigating Biological Systems Using Modeling: Strategies and Software. Academic Press, San Diego, CA 